\title{
ON A TAUBERIAN THEOREM BY O. SZÅSZ
}

\section{AMNON JAKIMOVSKI}

1. Renyi $[1]^{1}$ has proved that if the real series $\sum_{0}^{\infty} a_{n}$ is summable Abel to $s$, that is

(1.1) $\lim _{x \uparrow 1} \sum_{0}^{\infty} a_{n} x^{n}=\lim _{x \uparrow 1}(1-x) \cdot \sum_{0}^{\infty} s_{n} x^{n}=s \quad\left(s_{n}=a_{0}+\cdots+a_{n}\right)$ and

$$
\lim _{n \rightarrow \infty} \frac{\left|a_{1}\right|+2\left|a_{2}\right|+\cdots+n \cdot\left|a_{n}\right|}{n+1}=l<+\infty,
$$

then

$$
\sum_{0}^{\infty} a_{n}=s
$$

Later O. Szász [3] has given the following generalization of Renyi's theorem: From (1.1),

$$
\sum_{m=0}^{n} m \cdot\left(\left|a_{m}\right|-a_{m}\right)=O(n)
$$

and

$$
\lim _{m \rightarrow \infty} \frac{1}{m+1} \sum_{\nu=m+1}^{n} \nu \cdot\left(\left|a_{\nu}\right|-a_{\nu}\right)=0, \frac{n}{m} \rightarrow 1, n>m,
$$

or (1.1) and

$$
\begin{aligned}
\lim _{m \rightarrow \infty}\left\{\frac{1}{n} \cdot \sum_{\nu=0}^{n} \nu \cdot\left(\left|a_{\nu}\right|-a_{\nu}\right)-\frac{1}{m} \cdot \sum_{\nu=0}^{m} \nu \cdot\left(\left|a_{\nu}\right|-a_{\nu}\right)\right\} & =0, \\
\frac{n}{m} \rightarrow 1, n & >m,
\end{aligned}
$$

follow (1.2).

We shall see later that (1.3) is not necessary for the validity of the last theorem, and that (1.4), (1.4') may be replaced by more general conditions. We shall obtain a similar result for Borel's method of summability.

Received by the editors, September 2, 1952 and, in revised form, May 1, 1953.

1 Numbers in brackets refer to bibliography at the end of this note. 
Our main result will be

THEOREM 1. Necessary and sufficient conditions for the convergence of the real series $\sum_{0}^{\infty} a_{n}$ are (1.1) and one of the conditions

$$
\liminf _{m \rightarrow \infty} \frac{1}{m+1} \cdot \sum_{\nu=m+1}^{n} \nu a_{\nu} \geqq 0, \quad \frac{n}{m} \rightarrow 1, n>m,
$$

$\left(1.5^{\prime}\right) \quad \liminf _{m \rightarrow \infty}\left\{\frac{1}{n} \cdot \sum_{\nu=0}^{n} \nu a_{\nu}-\frac{1}{m} \cdot \sum_{\nu=0}^{m} \nu a_{\nu}\right\} \geqq 0, \quad \frac{n}{m} \rightarrow 1, n>m$.

It is easy to see that (1.5) includes (1.4) and that (1.5') includes (1.4').

Proof of Theorem 1. First we shall prove that (1.1), (1.5), and $\left(1.5^{\prime}\right)$ are necessary. From (1.2) follows (1.1) and

$$
t_{n}=\frac{s_{0}+s_{1}+\cdots+s_{n}}{n+1} \rightarrow s, \quad n \rightarrow \infty .
$$

Now

$$
t_{n}-t_{n-1}=\frac{1}{n} \cdot \frac{1}{n+1} \cdot \sum_{m=0}^{n} m a_{m}, \quad n \geqq 1,
$$

and

$$
\frac{1}{n} \cdot \sum_{\nu=0}^{n} \nu a_{\nu}=(n+1) \cdot\left(t_{n}-t_{n-1}\right)=s_{n}-t_{n}
$$

so that

$$
\lim _{n \rightarrow \infty} \frac{1}{n} \cdot \sum_{0}^{n} \nu a_{\nu}=0
$$

From this (1.5) and $\left(1.5^{\prime}\right)$ follow immediately.

We shall prove now that from (1.1) and (1.5) or (1.1) and $\left(1.5^{\prime}\right)$ follows (1.2).

It was proved by O. Szász [4] that from (1.1) follows

$$
\lim _{x \uparrow 1}(1-x) \cdot \sum_{n=0}^{\infty} t_{n} \cdot x^{n}=s .
$$

By subtracting (1.7) from (1.1) we get

$$
\lim _{x \uparrow 1}(1-x) \cdot \sum_{1}^{\infty} \frac{a_{1}+2 a_{2}+\cdots+n a_{n}}{n+1} \cdot x^{n}=0 .
$$

R. Schmidt has proved that if a real sequence $\left\{b_{n}\right\}$ is summable 
Abel and $\lim \inf _{m \rightarrow \infty}\left(b_{n}-b_{m}\right) \geqq 0, n / m \rightarrow 1, n>m$, then $\left\{b_{n}\right\}$ is convergent. From (1.7), Schmidt's theorem applied to the sequence $\left\{(1 / n) \cdot \sum_{\nu=0}^{n} \nu a_{v}\right\}$, and $\left(1.5^{\prime}\right)$ it follows that

$$
\lim _{n \rightarrow \infty} \frac{a_{1}+2 a_{2}+\cdots+n a_{n}}{n+1}=0,
$$

and by Tauber's classical theorem, (1.2) follows from (1.1) and (1.5'). We have

$$
s_{n}-s_{m}=\sum_{k=m+1}^{n} a_{k}=\sum_{k=m+1}^{n} \frac{k a_{k}}{k} .
$$

Abel's inequality yields

$$
s_{n}-s_{m} \geqq \frac{1}{m+1} \cdot \min _{m<k \leqq n} \sum_{\nu=m+1}^{k} \nu a,
$$

and (1.5) leads to

$$
\liminf _{m \rightarrow \infty}\left(s_{n}-s_{m}\right) \geqq 0, \quad \frac{n}{m} \rightarrow 1, n>m,
$$

thus we see, by Schmidt's theorem, that (1.2) follows from (1.1) and (1.5). Q.E.D.

2. A real series $\sum_{0}^{\infty} a_{n}$ is summable Borel to $s$ if

$$
\lim _{x \uparrow \infty} e^{-x} \cdot \sum_{0}^{\infty} \frac{s_{n}}{n !} \cdot x^{n}=s, \quad s_{n}=a_{0}+a_{1}+\cdots+a_{n} .
$$

It was proved by O. Szász [5] that from (2.1) follows

$$
\lim _{x \uparrow \infty} e^{-x} \cdot \sum_{0}^{\infty} \frac{t_{n}}{n !} \cdot x^{n}=s .
$$

By substracting (2.2) from (2.1) we obtain

$$
\lim _{x \uparrow \infty} e^{-x} \cdot \sum_{1}^{\infty} \frac{a_{1}+2 a_{2}+\cdots+n a_{n}}{n+1} \cdot \frac{x^{n}}{n !}=0 .
$$

Hardy and Littlewood have proved that if a series $\sum_{0}^{\infty} c_{n}$ is summable Borel and $c_{n}=o\left(1 / n^{1 / 2}\right)$, then $\sum_{0}^{\infty} c_{n}$ is convergent. By applying this theorem to the series $t_{0}+\sum_{n=1}^{\infty}\left(t_{n}-t_{n-1}\right)$ and using (2.2) we get

THEOREM 2. A necessary and sufficient condition for the convergence of $\sum_{0}^{\infty} a_{n}$ is that $\sum_{0}^{\infty} a_{n}$ should be summable Borel and that 


$$
\lim _{n \rightarrow \infty}\left(a_{1}+2 a_{2}+\cdots+n a_{n}\right) /(n+1)=0 .
$$

R. Schmidt [2] has proved that if the real series $\sum_{0}^{\infty} d_{n}$ is summable Borel and $\lim \inf _{m \rightarrow \infty}\left(d_{m+1}+\cdots+d_{n}\right) \geqq 0,(n-m) / m^{1 / 2} \rightarrow 0, n>m$, then $\sum_{0}^{\infty} d_{n}$ is convergent. By using this theorem, (2.3), and Theorem 2 we obtain

THEOREM 3. Necessary and sufficient conditions for the convergence of the real series $\sum_{0}^{\infty} a_{n}$ are (2.1) and one of the conditions

$$
\liminf _{m \rightarrow \infty} \frac{1}{m+1} \cdot \sum_{\nu=m+1}^{n} \nu a_{\nu} \geqq 0, \quad \frac{n-m}{m^{1 / 2}} \rightarrow 0, n>m .
$$

$$
\liminf _{m \rightarrow \infty}\left\{\frac{1}{n} \sum_{\nu=0}^{n} \nu a_{\nu}-\frac{1}{m} \sum_{\nu=0}^{m} \nu a_{\nu}\right\} \geqq 0, \quad \frac{n-m}{m^{1 / 2}} \rightarrow 0, n>m \text {. }
$$

\section{BIBLIOGRAPHY}

1. A. Renyi, Ona tauberian theorem of O.Szasz, Acta Univ. Szeged. Sect. Sci. Math. vol. 11 (1946) pp. 119-123.

2. R. Schmidt, Die umkehrsatze des Borelschen Summierungsverfahrens, Schriften der Königsberger Gelehrten Gesellschaft (1925).

3. O. Szász, On a tauberian theorem for Abel's summability, Pacific Journal of Mathematics vol. 1 (1951) pp. 117-125.

4. —_ Verallgemeinerung eines Littlewoodeschen Satzes über Potenzreihen, J. London Math. Soc. vol. 3 (1928).

5. - On products of summability methods, Proc. Amer. Math. Soc. vol. 3 (1952) pp. 257-262.

Tel-Aviv, IsRael. 